\title{
Spin-orbit coupling and phase-coherent transport in InN nanowires
}

\author{
G. Petersen, S. Estévez Hernández, R. Calarco, N. Demarina, ${ }^{*}$ and Th. Schäpers ${ }^{\dagger}$ \\ Institute of Bio- and Nanosystems (IBN-1) and JARA-Fundamentals of Future Information Technology, \\ Research Centre Jülich GmbH, 52425 Jülich, Germany
}

(Received 2 June 2009; published 18 September 2009)

\begin{abstract}
The low-temperature quantum transport properties of gated $\mathrm{InN}$ nanowires were investigated. Magneticfield-dependent as well as gate-dependent measurements of universal conductance fluctuations were performed to gain information on the phase coherence in the electron transport. We found a pronounced decrease in the variance of the conductance by about a factor of 2 in gate-dependent fluctuation measurements if a magnetic field is applied. This effect is explained by the suppression of the Cooperon channel of the electron correlation contributing to the conductance fluctuations. Despite the fact that the diameter of the nanowire is less than 100 $\mathrm{nm}$ a clear weak antilocalization effect is found in the averaged magnetoconductance being in strong contrast to the suppression of weak antilocalization for narrow quantum wires based on planar two-dimensional electron gases. The unexpected robustness of the weak antilocalization effect observed here is attributed to the tubular topology of the surface electron gas in InN nanowires.
\end{abstract}

DOI: 10.1103/PhysRevB.80.125321

PACS number(s): 73.23.- b, 72.15.Rn, 73.63.Nm

Semiconductor nanowires fabricated by a bottom-up approach are not only interesting for the realization of future nanoscaled devices ${ }^{1,2}$ but also appear to be very attractive model systems to tackle fundamental questions concerning the transport in strongly confined systems. ${ }^{3-5}$ In order to avoid the problem connected with carrier depletion, narrowband gap semiconductors, i.e., InAs or InN, ${ }^{1,6,7}$ are preferred. The underlying reason is that here the Fermi-level pinning in the conduction band results in a carrier accumulation at the surface. In fact, the tubular topology of the surface electron gas opens up the possibility to observe unconventional quantum transport phenomena. ${ }^{7}$ When the phase-coherence length $l_{\phi}$ in the nanowire is comparable to its dimensions the conductance fluctuates if a magnetic field is applied or if the electron concentration is changed by means of a gate electrode.$^{8-10}$ These so-called universal conductance fluctuations being in the order of $e^{2} / h$ originate from the fact that in small disordered samples, electron interference effects are not averaged out. ${ }^{11,12}$

Here, we analyzed universal conductance fluctuations to study the quantum transport properties in InN nanowires. In contrast to previous investigations ${ }^{6,7,9,10}$ the successful preparation of a top-gate electrode allowed us to study universal conductance fluctuations not only as a function of magnetic field but also as a function of gate voltage. Since $\mathrm{InN}$ is a narrow band gap semiconductor, one naturally expects spinorbit coupling effects similar to the case of InAs. ${ }^{13}$ Because this phenomena is of importance for spin electronic applications, we devoted special attention to the open question if spin-orbit coupling is present in InN nanowires. In transport measurements information on the spin-orbit coupling can be gained from the analysis of the characteristic beating pattern in Shubnikov-de Haas oscillations ${ }^{14}$ or by studying the weak antilocalization effect. ${ }^{15}$ However, owing to the cylindrical symmetry of the InN nanowires, in our case only the latter effect was observable and actually be used to determine the strength of spin-orbit coupling. In order to clearly separate the weak antilocalization effect from the conductance fluctuations, the averaging of the magnetoconductance at different gate voltages was essential.
The InN nanowires were grown without catalyst on a $\mathrm{Si}$ (111) substrate by plasma-assisted molecular beam epitaxy. After separating the wires from their original substrate, they were placed on a $\mathrm{SiO}_{2}$-covered $\mathrm{Si}$ (100) substrate patterned with contact pads and adjustment markers. The nanowires were contacted individually by a pair of Ti/Au electrodes. In order to control the electron concentration in the nanowires, $\mathrm{Cr} / \mathrm{Au}$ gate electrodes were placed in between the Ohmic contacts. Gate isolation was achieved by introducing a 30nm-thick $\mathrm{SiO}_{2}$ layer between the nanowire and the gate electrode.

The nanowire used here has a diameter $d$ of $85 \mathrm{~nm}$ and a total length of $1.2 \mu \mathrm{m}$. The distance $L$ between the Ohmic contacts is $300 \mathrm{~nm}$. A micrograph of the nanowire with Ohmic contacts and gate electrode is depicted in Fig. 2(a) (inset). At zero gate voltage the nanowire has a resistance of $2.0 \mathrm{k} \Omega$ after subtracting the typical contact resistance of $250 \Omega$. From measurements of the drain-source current as a function of gate voltage, an electron concentration $n_{2 d}$ of $7.7 \times 10^{13} \mathrm{~cm}^{-2}$ was determined from the extrapolated threshold gate voltage $V_{T h}=-170 \mathrm{~V} .{ }^{16}$ For the mobility and diffusion constant $\mathcal{D}$ we estimated a value of $46 \mathrm{~cm}^{2} / \mathrm{Vs}$ and $120 \mathrm{~cm}^{2} / \mathrm{s}$, respectively.

The magnetotransport measurements were performed in a He- 3 cryostat at temperatures down to $0.6 \mathrm{~K}$ and in a dilution refrigerator at temperatures between $4.2 \mathrm{~K}$ and $30 \mathrm{mK}$. The magnetoresistance was measured by using a lock-in technique with an ac bias current of $50 \mathrm{nA}$ and a magnetic field perpendicular to the wire axis.

Typical conduction fluctuation patterns for magnetic field sweeps at zero gate voltage $\left(V_{G}=0\right)$ and gate voltage sweeps at zero magnetic field $(B=0)$ are shown in Figs. 1(a) and 1(b), respectively. In both cases the fluctuation amplitude decreases with increasing temperatures. The mean fluctuation amplitude is quantified by the root mean square $(\mathrm{rms})$ of the conductance fluctuations $\operatorname{rms}(G)_{B}={\sqrt{\operatorname{var}(G)_{B}}}_{B}$ and $\operatorname{rms}(G)_{V_{G}}=\sqrt{\operatorname{var}(G)}_{V_{G}}$ over $B$ and $V_{G}$, respectively. Here, the variance of $G$ is defined by $\operatorname{var}(G)_{B, V_{G}}=\left\langle\delta G^{2}\right\rangle_{B, V_{G}}$, with $\langle\cdots\rangle_{B, V_{G}}$ the average of the conductance fluctuations $\delta G$ over $B$ and $V_{G}$, respectively. 


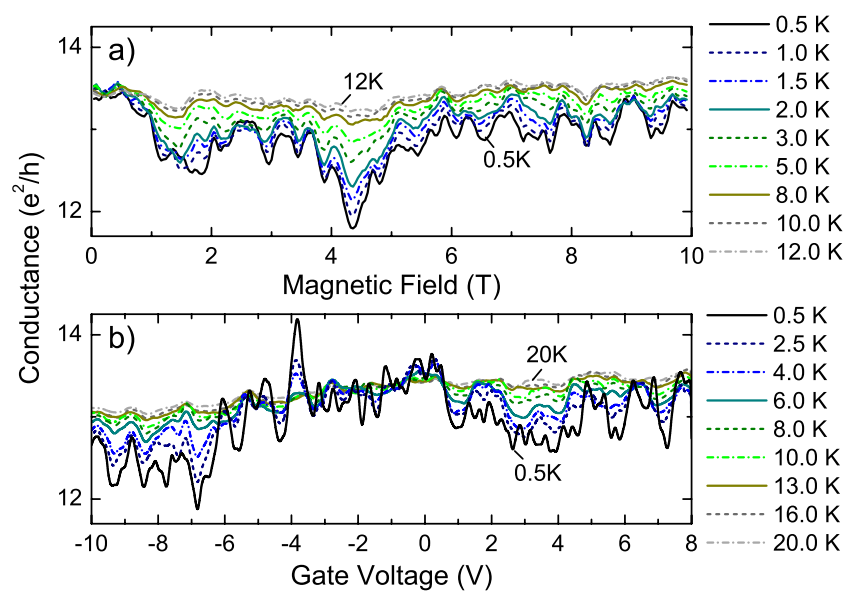

FIG. 1. (Color online) (a) Fluctuating conductance in units of $e^{2} / h$ as a function of magnetic field at various temperatures and $V_{G}=0$. (b) Corresponding measurements as a function of gate voltage at $B=0$.

As can be seen in Fig. 2(a), below about $1.5 \mathrm{~K} \mathrm{rms}(G)_{B, V_{G}}$ tends to saturate. Furthermore, the values of $\operatorname{rms}(G)_{V_{G}}$ are larger than the corresponding values of $\operatorname{rms}(G)_{B}$. In both cases the rms values are in the order of $e^{2} / h$ as predicted for universal conductance fluctuations. ${ }^{11,12}$ For larger temperatures a decrease of $\operatorname{rms}(G)_{B}$ and $\operatorname{rms}(G)_{V_{G}}$ proportional to $T^{-0.54}$ and $T^{-0.74}$ is found, respectively.

The corresponding conductance fluctuations were also characterized by the correlation field $B_{c}$ which is defined by $F_{B}\left(B_{c}\right)=\frac{1}{2} F_{B}(0)$. Here, $F_{B}(\Delta B)=\langle\delta G(B+\Delta B) \delta G(B)\rangle_{B}$ is the correlation function at $V_{G}=0$. As can be seen in Fig. 2(b), $B_{C}$ monotonically increases with $T^{0.13}$ in the whole temperature range. The phase-coherence length $l_{\phi}$ can be determined

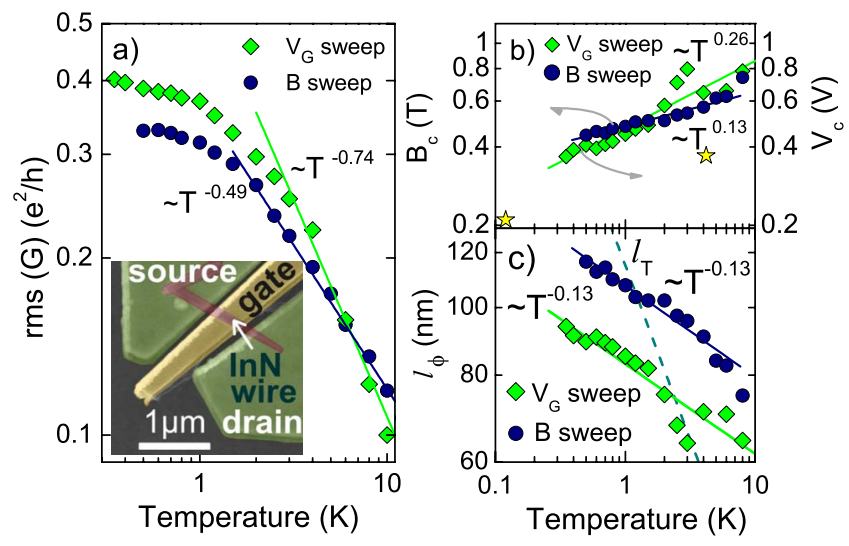

FIG. 2. (Color online) (a) rms of the conductance fluctuations determined from magnetic field sweep at $V_{G}=0$ and gate voltage sweep measurements at $B=0$ as a function of temperature. The inset shows an electron beam micrograph of the sample. (b) Correlation field $B_{c}$ and correlation voltage $V_{c}$ as a function of temperature. The solid lines correspond to the fits to the experimental data. The data points represented by $(\star)$ correspond to $B^{*}$ determined from the widths of the peaks in Fig. 3. (c) Phase-coherence length $l_{\phi}$ extracted from $B_{c}$ and $V_{c}$ as a function of temperature. The solid lines represent the fits to the experimental data. The dashed line represents the thermal length $l_{T}$.
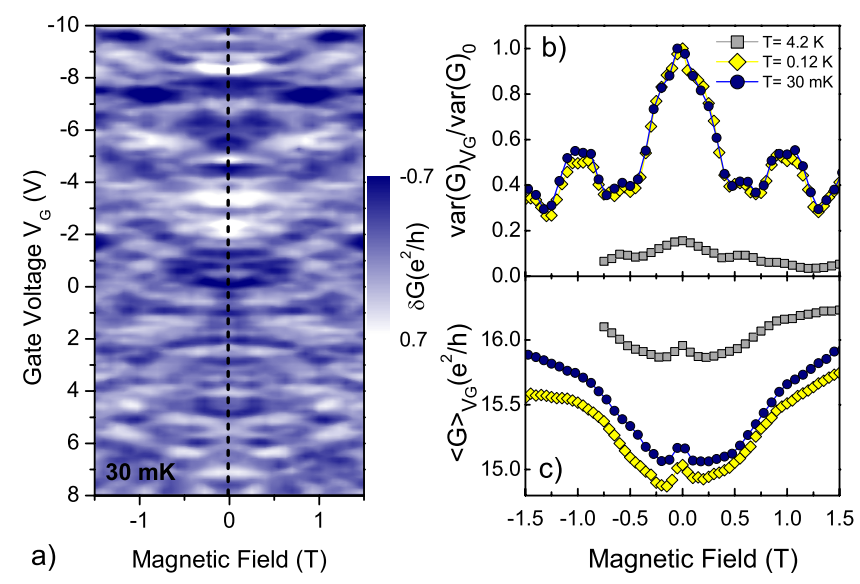

FIG. 3. (Color online) (a) Color scale plot of the conductance fluctuations $\delta G$ in units of $e^{2} / h$ as a function of $B$ and $V_{G}$ at $30 \mathrm{mK}$. (b) Normalized variance $\operatorname{var}(G)_{V_{G}} / \operatorname{var}(G)_{0}$ as a function of $B$ at 30 $\mathrm{mK}, 0.12$, and $4.2 \mathrm{~K}$. Here, $\operatorname{var}(G)_{0}$ is the variance at $B=0$ for the measurement at $30 \mathrm{mK}$. (c) Mean value of the conductance $\langle G\rangle_{V_{G}}$ in units of $e^{2} / h$ as a function of $B$ at $30 \mathrm{mK}, 0.12$, and $4.2 \mathrm{~K}$.

from $B_{c}$ by using the expression $l_{\phi} \approx \Phi_{0} / B_{c} d,{ }^{12}$ with $\Phi_{0}$ $=h / e$ the magnetic flux quantum. The resulting values of $l_{\phi}$ are shown in Fig. 2(c). In the whole temperature range $l_{\phi}$ is smaller than $L$, with $l_{\phi}=115 \mathrm{~nm}$ at $0.8 \mathrm{~K}$ being reduced to about $85 \mathrm{~nm}$ at $6 \mathrm{~K}$.

Similarly to $B_{c}$ the correlation voltage $V_{c}$ is defined by $F_{V_{G}}\left(V_{c}\right)=\frac{1}{2} F_{V_{G}}(0)$ with the correlation function at $B=0$ given by $F_{V_{G}}\left(\Delta V_{G}\right)=\left\langle\delta G\left(V_{G}+\Delta V_{G}\right) \delta G\left(V_{G}\right)\right\rangle_{V_{G}}{ }^{12}$ As can be seen in Fig. 2(b), $V_{c}$ increases with $T^{0.26}$. Since at higher temperatures the average conductance changes by less than $5 \%$ in the whole gate voltage range and since the majority of the carriers are located in the surface accumulation layer with a fixed distance to the gate electrode we can assume that $n_{2 d}$ changes linearly with $V_{G}$. Based on the fact that the Fermi energy is proportional to $n_{2 d}$ the following expression can be derived for $l_{\phi}$ as a function of the correlation voltage: $l_{\phi}$ $=\sqrt{2 m^{*} \mathcal{D} V_{T h} / \hbar n_{2 d} V_{c}}$. As can be inferred from Fig. 2(c), the values of $l_{\phi}$ decrease with the same slope as the corresponding values determined from $B_{c}$. Although the $l_{\phi}$ values obtained here are slightly lower compared to the ones calculated from $B_{c}$, one finds that generally both approaches lead to consistent results. As can be observed in Fig. 2(c), the thermal diffusion length $l_{T}$ falls below $l_{\phi}$ at a temperature around $2 \mathrm{~K}$. Thus, above that temperature an additional damping of the fluctuation pattern is expected. This is confirmed by the data shown in Fig. 2(a), where the onset of the stronger decrease of $\operatorname{rms}(G)$ is found around that temperature.

As mentioned above, in Fig. 2(a) one finds that at low temperatures $(T<5 \mathrm{~K}) \mathrm{rms}(G)_{V_{G}}$ exceeds $\operatorname{rms}(G)_{B}$. In order to study this phenomena in more detail, we measured the conductance fluctuations $\delta G$ as a function of $V_{G}$ at various magnetic fields at $30 \mathrm{mK}, 0.12$ and $4.2 \mathrm{~K}$, respectively. Figure 3(a) exemplarily shows $\delta G$ vs. $B$ and $V_{G}$ at $30 \mathrm{mK}$. Owing to the two-terminal measurement configuration $\delta G$ is symmetric with respect to $B$. As can be seen in Fig. 3(b), $\operatorname{var}(G)_{V_{G}}$ shows a clear peak at $B=0$ for all measured tem- 
peratures. In accordance to the results shown in Fig. 2(a), $\operatorname{rms}(G)_{V_{G}}$ is substantially reduced at $4.2 \mathrm{~K}$ compared to the measurements at lower temperatures. As expected from theory, ${ }^{17,18}$ the application of a sufficiently large magnetic field reduces $\operatorname{var}(G)_{V_{G}}$ to approximately one-half of the value at zero field. A similar behavior was previously observed, e.g., in GaAs/AlGaAs or Li wires. ${ }^{19,20}$ From a fit to a quadratic Lorentzian curve, we determined a half-width at halfmaximum of $B^{*}=0.21 \mathrm{~T}$ for the measurements at $30 \mathrm{mK}$ and $0.12 \mathrm{~K}$, while for the measurement at $4.2 \mathrm{~K}$ we obtained a value of $0.35 \mathrm{~T}$. Theoretically it is expected that $B^{*}$ corresponds to the correlation field $B_{c}$ defined above. ${ }^{17,18}$ As can be seen in Fig. 2(b), the $B^{*}$ values determined from the peak width are slightly below the $B_{c}$ values previously obtained.

Next we will turn to discussion of weak (anti)localization effects which can be accessed by averaging the conductance over the gate voltage $\langle G\rangle_{V_{G}}$ and thus suppressing the conductance fluctuations. The weak localization effect indicated by an increased resistance originates from the interference of coherent time-reversed electron paths. In the presence of spin-orbit coupling the additional contribution due to spin precession results in a net decrease of the resistance. Therefore this effect is called antilocalization. In Fig. 3(c) $\langle G\rangle_{V_{G}}$ is plotted as a function of $B$ for $30 \mathrm{mK}, 0.12$, and $4.2 \mathrm{~K}$, respectively. As can be seen here, by averaging the gatevoltage sweeps of the conductance the fluctuating contribution is eliminated completely. For all temperatures we find a broad minimum at $B=0$ with a width of approximately $2 \mathrm{~T}$, which we attributed to the weak localization effect. ${ }^{21} \mathrm{~A}$ closer look reveals that in the center of this minimum a peak appears, which can be assigned to the weak antilocalization effect due to the presence of spin-orbit coupling. ${ }^{15}$

The assumption that spin-orbit coupling is present is also supported by the measurements shown in Fig. 3(b). Only a single drop of $\operatorname{var}(G)_{B, V_{G}}$ to about half of the initial value is observed when the magnetic field is increased. The appearance of universal conductance fluctuations can be explained by electron correlations which can be subdivided in the socalled Cooperon and diffusion channels. ${ }^{17,18}$ The presence of spin-orbit coupling suppresses the triplet contribution in both channels, resulting in a reduction of the variance by a factor of 4 at $B=0$. In fact, as can be seen in Fig. 2(a), the low temperature $\operatorname{rms}(G)_{V_{G}}$ value of $\approx 0.4 e^{2} / h$ for $B=0$ is very close to the theoretically expected value of $0.43 .{ }^{12,18}$ Applying a magnetic field results in a complete suppression of the Cooperon channel owing to breaking of time-reversal symmetry. This leads to the observed decrease of $\operatorname{var}(G)$ by onehalf.

In order to investigate the weak antilocalization effect in more detail, additional measurements with a higher magnetic field resolution were performed. As can be seen in Fig. 4, the height of the conductance peak decreases by about a factor of 2 , when the temperature is increased from 0.5 to $4.0 \mathrm{~K}$. By fitting the experimental data points to the Kettemann model, ${ }^{22}$ which is the appropriate model for wire structures, the phase-coherence length $l_{\phi}$ and spin-relaxation length $l_{s o}$ were extracted. In Fig. 4 the corresponding fitted conductance corrections are shown. We found that a satisfying fit is obtained by keeping $l_{s o}$ constant at $63 \mathrm{~nm}$ while $l_{\phi}$ was

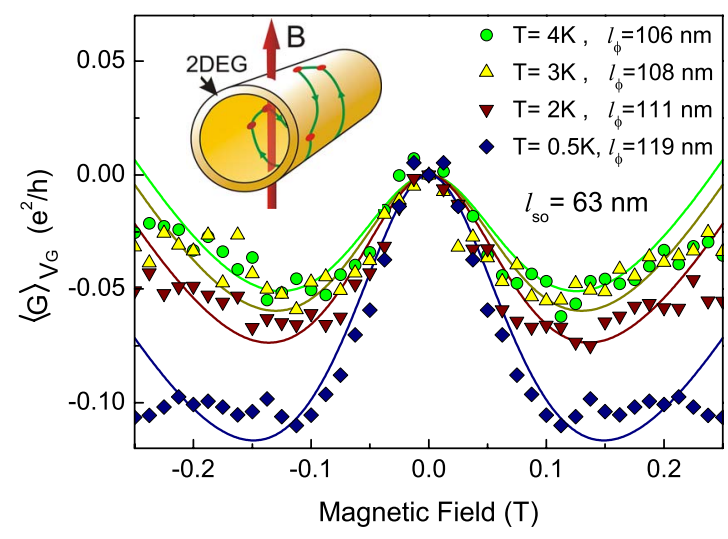

FIG. 4. (Color online) Mean value of the conductance $\langle G\rangle_{V_{G}}$ in units of $e^{2} / h$ as a function of $B$ at temperatures of $0.5,2.0,3.0$, and $3.0 \mathrm{~K}$, respectively. The solid lines represent the fits based on the Kettemann model (Ref. 22). The inset shows a schematics of a closed electron trajectory in the surface electron gas of the $\mathrm{InN}$ wire.

gradually decreased from $119 \mathrm{~nm}$ at $0.5 \mathrm{~K}$ to $106 \mathrm{~nm}$ at 4.0 $\mathrm{K}$. The values of $l_{\phi}$ are close to the values shown in Fig. 2, which were obtained from the correlation field.

Compared to other material systems ${ }^{23,24}$ the spin-orbit scattering length $l_{s o}$ obtained from the fit is rather small and consequently ends up in a large spin-orbit coupling parameter $\alpha$. The latter can be calculated by $\alpha=\hbar^{2} /\left(2 m^{*} l_{\text {so }}\right)$ resulting in $8.6 \times 10^{-12} \mathrm{eV} \mathrm{m}$ if one assumes an effective mass of $m^{*}=0.07 m_{e} \cdot{ }^{25}$ A possible origin of spin-orbit coupling is the Rashba effect due to the electric field in the surface electron gas. ${ }^{26}$ We estimated the theoretically expected Rashba coupling parameter $\alpha$ by assuming that the electrons are located predominantly in a surface two-dimensional electron gas (2DEG). ${ }^{7}$ The wave function and potential profile of a cylindrical 2DEG required for the calculation of the Rashba coupling parameter ${ }^{27}$ was gained from a Schrödinger-Poisson solver. In contrast to the value resulting from the fit to the Kettemann model, we obtained considerably smaller value of $0.6 \times 10^{-12} \mathrm{eV} \mathrm{m}$ for $\alpha_{R}$. We attribute the small value to the fact that although $\mathrm{InN}$ has a narrow band gap $^{25}\left(E_{g}\right.$ $=0.78 \mathrm{eV}$ ) being in principle advantageous for the Rashba effect, the spin-orbit splitting in the valence band is very small $\left(\Delta_{\text {so }} \approx 5 \mathrm{meV}\right) .{ }^{25}$ The latter causes the Rashba effect to be reduced. ${ }^{27}$

The large discrepancy between the spin-orbit coupling parameter obtained from the fit to the Kettemann model and to the value resulting from the band profile might be due to the following reasons. First, in our calculation of $\alpha$ only the Rashba contribution was considered, while the contribution due to the bulk inversion asymmetry ${ }^{28}$ was disregarded since no data on its strength is available yet. Thus the total strength of theoretically expected spin-orbit coupling might be higher than the value estimated here. Second, the topology of a tubelike conductor can lead to a flux cancellation effect. As illustrated in Fig. 4 (inset), some closed loops contributing to the weak antilocalization effect partially cover the upper and lower part of the tubelike conductor. Due to the resulting reversed orientations with respect to the vector potential a flux cancellation effect is expected. This flux cancellation 
should result in a broadening of the weak antilocalization peak and consequently to an overestimated value of $\alpha$ in the fit to the Kettemann model. This probably also explains why the spin-orbit scattering time $\tau_{s o}=l_{s o}^{2} / \mathcal{D}$ of $0.33 \times 10^{-12} \mathrm{~s}$ obtained in our experiment is lower than the value of 1.3 $\times 10^{-12} \mathrm{~s}$ found by Jia et al. ${ }^{29}$ for InN thin films. Remarkably, in our nanowires no suppression of weak antilocalization is observed as it was found in narrow wires based on planar two-dimensional electron gases. ${ }^{30} \mathrm{We}$ attribute this to the fact that in our case due to the tubular topology no diffusive boundary scattering can occurs. The latter effect is responsible for the suppression of the weak antilocalization in planar wires.

In conclusion, we could show that the conductance fluctuations in $\mathrm{InN}$ nanowires measured as a function of gate voltage or magnetic field show very similar temperature de- pendencies. From the correlation field $B_{c}$ the phasecoherence length was estimated to be up to $115 \mathrm{~nm}$ at $0.8 \mathrm{~K}$. A clear signature of the presence of spin-orbit coupling was found, which is an important prerequisite for incorporating InN nanowires in future spin electronic device structures. However, since the available theoretical models for the weak antilocalization effect in quantum wires do not account for the special tubular topology in our wires, the extracted values for the spin-orbit coupling strength are probably overestimated. We hope that our results initiate further theoretical studies which properly describe the weak antilocalization effect in tubular conductors.

The authors thank U. Zülicke (Massey University, New Zealand) as well as J. König and M. Governale (University Duisburg-Essen, Germany) for fruitful discussions.
*Permanent address: Electronics Department, Radiophysics Faculty, Nizhny Novgorod State University, Nizhny Novgorod 603950, Russia.

${ }^{\dagger}$ th.schaepers@fz-juelich.de

${ }^{1}$ C. Thelander et al., Mater. Today 9, 28 (2006).

${ }^{2}$ W. Lu and C. M. Lieber, J. Phys. D 39, R387 (2006).

${ }^{3}$ S. De Franceschi, J. A. van Dam, E. P. A. M. Bakkers, L. Feiner, L. Gurevich, and L. P. Kouwenhoven, Appl. Phys. Lett. 83, 344 (2003).

${ }^{4}$ C. Fasth, A. Fuhrer, M. T. Bjork, and L. Samuelson, Nano Lett. 5, 1487 (2005).

${ }^{5}$ A. Pfund, I. Shorubalko, R. Leturcq, and K. Ensslin, Appl. Phys. Lett. 89, 252106 (2006).

${ }^{6}$ C.-Y. Chang, G.-C. Chi, W.-M. Wang, L.-C. Chen, K.-H. Chen, F. Ren, and S. J. Pearton, Appl. Phys. Lett. 87, 093112 (2005).

${ }^{7}$ T. Richter, Ch. Blömers, H. Lüth, R. Calarco, M. Indlekofer, M. Marso, and Th. Schäpers, Nano Lett. 8, 2834 (2008).

${ }^{8}$ A. E. Hansen, M. T. Björk, C. Fasth, C. Thelander, and L. Samuelson, Phys. Rev. B 71, 205328 (2005).

${ }^{9}$ Ch. Blömers, Th. Schäpers, T. Richter, R. Calarco, H. Lüth, and M. Marso, Appl. Phys. Lett. 92, 132101 (2008).

${ }^{10}$ Ch. Blömers, Th. Schäpers, T. Richter, R. Calarco, H. Lüth, and M. Marso, Phys. Rev. B 77, 201301(R) (2008).

${ }^{11}$ B. Al'tshuler, Pis'ma Zh. Eksp. Teor. Fiz. 41, 530 (1985) [JETP Lett. 41, 648 (1985)].

${ }^{12}$ P. A. Lee, A. D. Stone, and H. Fukuyama, Phys. Rev. B 35, 1039 (1987).

${ }^{13}$ C. Schierholz, T. Matsuyama, U. Merkt, and G. Meier, Phys. Rev. B 70, 233311 (2004)

${ }^{14}$ J. Luo, H. Munekata, F. F. Fang, and P. J. Stiles, Phys. Rev. B 38, 10142 (1988).
${ }^{15}$ S. Hikami, A. I. Larkin, and Y. Nagaoka, Prog. Theor. Phys. 63, 707 (1980).

${ }^{16}$ G. Cheng, E. Stern, D. Turner-Evans, and M. A. Reed, Appl. Phys. Lett. 87, 253103 (2005).

${ }^{17}$ B. Al'tshuler and B. Shklovskii, Zh. Eksp. Teor. Fiz. 91, 220 (1985) [Sov. Phys. JETP 64, 127 (1986)].

${ }^{18}$ A. D. Stone, Phys. Rev. B 39, 10736 (1989).

${ }^{19}$ P. Debray, J.-L. Pichard, J. Vicente, and P. N. Tung, Phys. Rev. Lett. 63, 2264 (1989).

${ }^{20}$ J. S. Moon, N. O. Birge, and B. Golding, Phys. Rev. B 53, R4193 (1996).

${ }^{21}$ C. W. J. Beenakker and H. van Houten, in Solid State Physics, edited by H. Ehrenreich and D. Turnbull (Academic, New York, 1991), Vol. 44, p. 1.

${ }^{22}$ S. Kettemann, Phys. Rev. Lett. 98, 176808 (2007).

${ }^{23}$ J. B. Miller, D. M. Zumbühl, C. M. Marcus, Y. B. LyandaGeller, D. Goldhaber-Gordon, K. Campman, and A. C. Gossard, Phys. Rev. Lett. 90, 076807 (2003).

${ }^{24}$ V. A. Guzenko, Th. Schäpers, and H. Hardtdegen, Phys. Rev. B 76, 165301 (2007).

${ }^{25}$ I. Vurgaftman and J. R. Meyer, J. Appl. Phys. 94, 3675 (2003).

${ }^{26}$ Yu. A. Bychkov and E. I. Rashba, J. Phys. C 17, 6039 (1984).

${ }^{27}$ Th. Schäpers, G. Engels, J. Lange, Th. Klocke, M. Hollfelder, and H. Lüth, J. Appl. Phys. 83, 4324 (1998).

${ }^{28}$ E. I. Rashba, Fiz. Tverd. Tela (Leningrad) 2, 1224 (1960) [Sov. Phys. Solid State 2, 1109 (1960)].

${ }^{29}$ Z. W. Jia, W. Z. Shen, H. Ogawa, and Q. X. Guo, Appl. Phys. Lett. 89, 232107 (2006).

${ }^{30}$ Th. Schäpers, V. A. Guzenko, M. G. Pala, U. Zülicke, M. Governale, J. Knobbe, and H. Hardtdegen, Phys. Rev. B 74, 081301(R) (2006). 